\title{
Aplicação de índice de estresse para a tolerância ao frio no desenvolvimento inicial de cultivares de arroz
}

\section{Application of stress indexes to evaluate cold tolerance of rice cultivars during the initial stage of plant development}

Hélen Claudine Saliba RODRIGUES ${ }^{1}$; Caroline Borges BEVILACQUA ${ }^{2}$; Carlos André BAHRY ${ }^{2}$; Daisy Letícia Ramirez MONZON ${ }^{3}$; Taiane Peres VIANA ${ }^{4}$; Paulo Dejalma ZIMMER Paulo Ricardo Reis FAGUNDES ${ }^{6}$

${ }^{1}$ Parte da dissertação de mestrado do primeiro autor; Pesquisa financiada pela Coordenação de Aperfeiçoamento de Pessoal de Nível Superior - CAPES. Autor para correspondência; Biológa, Mestre em Ciência e Tecnologia de Sementes; Departamento de Fitotecnia, Universidade Federal de Pelotas (UFPel); Laboratório de Análises de Sementes Flávio Rocha.; Caixa Postal:354, CEP 96001-970, Pelotas, RS, Brasil. completo da instituição; helensaliba@gmail.com

${ }^{2}$ Doutor em Ciência e Tecnologia de Sementes; Departamento de Fitotecnia, Universidade Federal de Pelotas; carolinebevi@gmail.com; carlosbahry@hotmail.com

${ }^{3}$ Mestre em Ciência e Tecnologia de Sementes; Departamento de Fitotecnia, Universidade Federal de Pelotas; daisyleti@hotmail.com

${ }^{4}$ Mestre em Agronomia; Departamento de Fitotecnia, Universidade Federal de Pelotas; taipv_@hotmail.com

5 Eng. Agr., Dr. Professor da Universidade Federal de Pelotas (UFPel). djzimmer@ufpel.edu.br

${ }^{6}$ Eng. Agr.,Dr. Pesquisador da Embrapa Clima Temperado - CPACT. Paulo.fagundes@cpact.embrapa.br

Recebido em: 01-06-2013; Aceito em: 28-04-2014

\section{Resumo}

O estresse por frio tem forte impacto no desenvolvimento, reprodução e distribuição de plantas no Brasil. As baixas temperaturas registradas no Rio Grande do Sul são um dos principais fatores que limitam o rendimento das plantas de origem tropical, como o arroz. Geralmente, estas plantas são sensíveis ao estresse por frio, o que torna a busca pela tolerância a esse fator uma necessidade na fase de desenvolvimento. Por isso, este estudo teve por objetivo verificar se os índices de tolerância ao frio, índices de suscetibilidade ao frio e média geométrica são eficientes para avaliar a tolerância ao frio das cultivares de arroz irrigado Brilhante, BRS Querência e SCSBRS Tio Taka, na fase de desenvolvimento inicial. $O$ índice de tolerância ao estresse, índice de suscetibilidade ao estresse e média geométrica foram utilizados para avaliar o desempenho das cultivares em condições de baixa temperatura. Os índices de velocidade de desenvolvimento inicial e os índices dos estádios V1, V2 e V3 de cada genótipo, em ambiente com estresse, em BOD a temperatura alternada $\left(13^{\circ} \mathrm{C}\right.$ por $10 \mathrm{~h} \mathrm{e}$ $18^{\circ} \mathrm{C}$ por $14 \mathrm{~h}$ ) e em ambiente sem estresse (casa de vegetação- $25^{\circ} \mathrm{C}$ ) foram utilizados para calcular os índices de estresses. Os resultados mostraram que foi possível classificar o desempenho das cultivares baseado nesses índices de estresse em condições de baixa temperatura. Os índices de estresse avaliados são eficientes para estimar a tolerância das cultivares de arroz em condições de baixa temperatura, destacando-se a superioridade da cultivar Brilhante nas fases iniciais de desenvolvimento (S0-V3) da cultura do arroz no Rio Grande do Sul.

Palavras-chave adicionais: baixa temperatura; índice de velocidade de desenvolvimento; média geométrica.

\begin{abstract}
Cold stress has a strong impact on development, reproduction, and plant distribution in Brazil. The low temperatures usually registered in the state of Rio Grande do Sul is one of the most important factors limiting yields of plants of tropical origin such as is the case of rice. Usually these plants are sensitive to cold stress so that the search for cold tolerant cultivars is of necessity. This is why this study viewed to evaluate whether the cold tolerance indexes, cold susceptibility indexes and the geometric mean are efficient to evaluate cold tolerance of the irrigated rice cultivars 'Brilhante', 'BRS Querência', and SCSBRS Tio Taka' at the plants seedling stage. The speed indexes of the initial stage of development and the indexes of the stages $\mathrm{V} 1, \mathrm{~V} 2$, and $\mathrm{V} 3$ of each genotype in a stressed environment, in BOD under alternating temperature $\left(13^{\circ} \mathrm{C}\right.$ for 10 hours and $18^{\circ} \mathrm{C}$ for 14 hours) and in a non stressed environment (greenhouse at $25^{\circ} \mathrm{C}$ ) were used to calculate the stress indexes. The results showed that the used stress indexes permitted to rank the cultivars as to cold tolerance. Based on the results, cultivar 'Brilhante' was verified to be the most cold tolerant of the analyzed cultivars.
\end{abstract}

Additional keywords: geometric mean; low temperature; speed development index. 


\section{Introdução}

As baixas temperaturas do ar ou estresse por frio têm grande impacto no desenvolvimento, reprodução e distribuição de plantas. As plantas são caracterizadas pelo nível intrínseco de tolerância a baixas temperaturas (BEVILACQUA et al., 2013).

O estresse por frio pode reduzir a taxa metabólica, pois o cloroplasto é diretamente afetado pela baixa temperatura, causando danos ao aparelho fotossintético e à molécula de clorofila (STHAPIT et al., 1995). Na fase de desenvolvimento vegetativo, o frio provoca redução no estabelecimento de plantas, taxa de desenvolvimento diário das folhas, perfilhamento, altura das plantas e baixa capacidade de competição do arroz em relação às plantas daninhas (FREITAS et al., 2008).

A baixa temperatura no período reprodutivo pode prejudicar o desenvolvimento dos grãos de pólen, resultando em alta esterilidade de espiguetas, comprometendo a produtividade (LARROSA et al., 2009). O estresse por frio, verificado no Rio Grande do Sul, é um dos principais fatores que limitam o rendimento da cultura, pois plantas de origem tropical, como o arroz, apresentam sensibilidade ao frio. A tolerância ao frio varia conforme o período de desenvolvimento da planta (CRUZ, 2001), tornando a busca pela tolerância uma necessidade na fase de desenvolvimento inicial e no período crítico da cultura.

A seleção de cultivares tolerantes a baixas temperaturas no período de desenvolvimento inicial é difícil, devido à complexa base genética do caráter e à falta de controle do estresse em condições de campo (CRUZ, 2001). Dessa forma, foram desenvolvidos os índices de estresse para auxiliar no controle do estresse em ambientes. Estes índices têm sido utilizados em trabalhos realizados por PORCH et al. (2010), para avaliar o rendimento na cultura do feijão $\mathrm{e}$ por BEVILACQUA et al. (2013), ao compararem o desempenho de cultivares de arroz em condições de baixas temperaturas.. A média geométrica (MG) e o índice de tolerância ao estresse desenvolvido por FERNANDEZ (1993), e o índice de suscetibilidade ao estresse (FISHER \& MAURER, 1978) têm sido utilizados para comparar o desempenho de cultivares através dos anos em ambientes com e sem estresse.

A busca pela tolerância ao frio em cultivares de arroz, na fase de germinação e no desenvolvimento inicial, é de suma importância para a região Sul do Brasil, pois o uso de cultivares tolerantes ao frio possibilita a antecipação da semeadura em regiões onde o frio se torna um problema para essa cultura e evita que a fase reprodutiva coincida com a época de frio e ocorra no período de maior intensidade de radiação solar (MARTINS et al., 2007; MERTZ et al., 2009), potencializando a fotossíntese. Assim, este estudo teve como objetivo verificar se o índice de tolerância e o de suscetibilidade ao frio, assim como a média geométrica, são eficientes para avaliar a tolerância ao frio das cultivares de arroz irrigado Brilhante, BRS Querência e SCSBRS Tio Taka, na fase de desenvolvimento inicial (S0-V3).

\section{Material e métodos}

Foram escolhidas três cultivares de arroz irrigado com diferentes níveis de tolerância: BRS Querência (tolerante ao frio) e SCSBRS Tio Taka (sensível ao frio), registradas e recomendadas para a semeadura do arroz no Rio Grande do Sul (ARROZ IRRIGADO, 2010), e a cultivar Brilhante (origem chilena), que compõem 0 banco de germoplasma da Embrapa Clima Temperado, considerada tolerante ao frio, razão pela qual foi utilizada. As sementes foram tratadas com o produto Maxim + Thiram ${ }^{\circledR}$ e semeadas em copos de plástico de $200 \mathrm{~mL}$. O substrato utilizado foi solo do tipo planossolo, coletado em área de várzea da Embrapa Clima Temperado, no município de Capão do Leão (RS). Posteriormente, o solo foi esterilizado a $121^{\circ} \mathrm{C}$ por 1 hora, durante dois dias consecutivos, com o intuito de eliminar os microrganismos do solo (KENNEDY et al., 2011). O experimento foi conduzido em dois ambientes: com estresse por frio $\left(13^{\circ} \mathrm{C}\right.$ por $10 \mathrm{~h}$ e $18{ }^{\circ} \mathrm{C}$ por $14 \mathrm{~h}$ em BOD) e sem estresse (casa de vegetação a $25^{\circ} \mathrm{C}$ ). As cultivares foram avaliadas por meio dos seguintes parâmetros:

IVDi - Índice de velocidade de desenvolvimento inicial até o estádio $\mathrm{V} 3$

IVD V1 - índice de velocidade de desenvolvimento do estádio V1;

IVD V2 - índice de velocidade de desenvolvimento do estádio V2;

IVD V3 - índice de velocidade de desenvolvimento do estádio V3;

ITS - índice de tolerância ao estresse por frio;

ISS - índice de suscetibilidade ao estresse por frio;

MG - média geométrica (significância dos resultados).

Os índices de velocidade de desenvolvimento inicial (IVDi) e os índices de velocidade de desenvolvimento dos estádios IVD V1, IVD V2 e IVD V3, em ambiente com e sem estresse, foram calculados pela fórmula de MAGUIRE (1962): IVD $=D_{1} / N_{1}+D_{2} / N_{2}+\ldots+D_{n} / N_{n}$, em que: $D 1$, $\mathrm{D} 2, \mathrm{Dn}=$ número de plântulas desenvolvidas na primeira, segunda, até a última contagem; N1, $\mathrm{N} 2, \mathrm{Nn}=$ número de semanas desde a primeira, segunda, até a última contagem. 
Esses índices foram utilizados para calcular os seguintes índices de estresses: Índice de tolerância ao estresse por frio (ITS=(Yp $\times$ Ys) $\left./ \mathrm{Xp}^{2}\right)$; índice de suscetibilidade ao estresse por frio $(I S S=(1-(Y s / Y p)) /(1-(X s / X p)))$, proposto por FISCHER \& MAURER (1978) e média geométrica $M G=(Y s \times Y p)^{1 / 2} \quad(F E R N A N D E Z$, 1993), em que:

Ys: corresponde à média total do IVDi e IVD dos estádios V1, V2 e V3, para cada uma das cultivares em ambiente com estresse (BOD);

Yp: corresponde à média total do IVDi e IVD dos estádios V1, V2 e V3, para cada uma das cultivares em ambiente sem estresse (casa de vegetação);

Xs: corresponde à média total do IVDi e IVD dos estádios V1, V2 e V3, de todas as cultivares em ambiente com estresse (BOD);

$\mathrm{Xp}$ : corresponde à média total do IVDi e IVD dos estádios V1, V2 e V3, de todas as cultivares em ambiente sem estresse por frio (casa de vegetação).

O ranqueamento dos genótipos baseouse nos valores superiores dos índices de tolerância ao estresse e na média geométrica para cada cultivar. Este procedimento foi adotado com vistas a definir níveis de tolerância de cada cultivar nos estádios de desenvolvimento avaliados pelo índice de velocidade de desenvolvimento inicial e pelos índices de desenvolvimento de cada estádio vegetativo. Os dados dos índices de velocidade de desenvolvimento foram submetidos à análise de variância, e as médias, comparadas pelo teste de Tukey, a $5 \%$ de probabilidade. $O$ delineamento experimental utilizado na casa de vegetação e em BOD foi o inteiramente casualizado, com cinco repetições. Para análise da correlação linear de Pearson, foi utilizado o teste $\mathrm{t}$, a $1 \%$ e $5 \%$ de probabilidade.

\section{Resultados e discussão}

O índice de velocidade de desenvolvimento inicial (IVDi) foi sensível para estratificar as cultivares entre si, dentro de cada ambiente e entre os diferentes ambientes (com e sem estresse por frio) (Tabela 1).

Tabela 1 - Índice de velocidade de desenvolvimento inicial (IVDi)* de plantas de arroz em ambiente sem e com estresse por frio. Initial development speed index (IVDi) of rice plants in cold stressed and non-stressed environments.

\begin{tabular}{lccc}
\hline Cultivares & Sem estresse & Com estresse & ${ }^{(1)}$ Redução do IVDi (\%) \\
\hline Brilhante & $10,39 \mathrm{aA}$ & $5,31 \mathrm{aB}$ & 49 \\
SCSBRS Tio Taka & $8,45 \mathrm{bA}$ & $2,42 \mathrm{bB}$ & 71 \\
BRS Querência & $7,98 \mathrm{bA}$ & $2,54 \mathrm{bB}$ & 68 \\
\hline CV $(\%)$ & & 12,5 &
\end{tabular}

${ }^{*}$ Médias seguidas pela mesma letra maiúscula na linha e minúscula na coluna não diferem significativamente entre si, pelo teste de Tukey, ao nível de $5 \%$ de probabilidade. ${ }^{(1)}$ redução IVDi $(\%)=(($ valor com estresse/valor sem estresse $\left.)-1)\right) \times 100 ; \mathrm{CV}$ - coeficiente de variação.

A cultivar Brilhante apresentou maior índice de velocidade de desenvolvimento inicial em relação às outras duas cultivares, independentemente das condições de estresse. Quando comparado o efeito do ambiente (sem e com estresse por frio) em cada cultivar, foi possível verificar que as três cultivares apresentaram redução significativa no IVDi, quando submetidas ao estresse por frio. Nessa condição de estresse, as cultivares Brilhante, SCSBRS Tio Taka e BRS Querência apresentaram redução de 49\%, 71\% e $68 \%$, respectivamente, no IVDi, quando comparadas ao ambiente sem estresse. Isso evidencia que a cultivar Brilhante foi mais tolerante ao frio, apresentando melhor desempenho em relação às outras duas que, por sua vez, não apresentaram grande variação entre si. A cultivar Brilhante é de origem japônica, o que pode ter contribuído para que ela não apresentasse redução acentuada em seu IVDi, quando submetida ao frio, em comparação às outras cultivares do grupo japônico tropical, originadas de cruza- mentos múltiplos, ou seja, do cruzamento entre espécies índicas e japônicas. Por ser do grupo japônico, a cultivar Brilhante pode apresentar caracteres morfofisiológicos, como tolerância à descoloração foliar e maior estatura das plantas nas fases iniciais de desenvolvimento, que contribuam para a tolerância ao frio em arroz, passíveis de serem utilizadas como fonte em cruzamentos nos programas de melhoramento genético, visando a amenizar o problema "frio" para essa cultura no Rio Grande do Sul. Em trabalhos similares, MERTZ et al. (2009) conseguiram classificar as cultivares com relação à tolerância ao frio na fase de germinação, destacando maior tolerância para os genótipos pertencentes às subespécies japônicas em relação às de origem índica. Os mesmos autores observaram, em trabalhos similares, grande variabilidade de respostas ao estresse por frio entre cultivares japônicas e índicas, prevalecendo maior tolerância ao frio por parte das cultivares do grupo japônico. 
O desempenho das cultivares avaliadas pelo índice de velocidade de desenvolvimento até atingir o estádio V1 (Tabela 2) foi semelhante ao obtido para o IVDi (Tabela 1). O melhor desempenho da cultivar Brilhante também foi verificado no IVD em V2, tanto em ambiente com, como sem estresse por frio, não diferindo, porém, da SCSBRS Tio Taka no ambiente sem estresse. No IVD em V3, no ambiente sem estresse, não foi possível verificar diferença significativa entre as cultivares. No entanto, quando elas foram submetidas ao frio, novamente a cultivar Brilhante foi superior e não houve diferença entre a SCSBRS Tio Taka e a BRS Querência.

A avaliação do IVD em V1, V2 e V3, em cada cultivar, comparando os ambientes, mostrou resultado semelhante ao observado no IVDi, ou seja, redução na velocidade de desenvolvimento das plantas quando submetidas ao estresse por frio. Os valores percentuais obtidos na cultivar Brilhante foram proporcionais aos observados no IVDi para cada genótipo, quando comparados os distintos ambientes, evidenciando que temperaturas baixas comprometem $o$ índice de velocidade de desenvolvimento do arroz, ressaltando a superioridade da Brilhante. Esses resultados ratificam as afirmações feitas por STEINMETZ et al. (2008), que para o arroz obter uma emergência rápida e uniforme, a temperatura do solo deve ser igual ou superior a $20 \stackrel{\circ}{ }$ C. Outros resultados também foram encontrados pelos mesmos autores, ao relatarem que a fase vegetativa é muito influenciada pelas temperaturas do ar e do solo, pois a ocorrência de temperaturas entre $13^{\circ} \mathrm{C}$ e $18^{\circ} \mathrm{C}$ pode provocar aumento no ciclo das plantas, retardando a emergência dessas em mais de 20 dias. Isso ocorre, principalmente, em semeaduras realizadas no mês de setembro, no Rio Grande do Sul, e também devido ao uso de cultivares sensíveis, prejudicando assim a taxa de crescimento na fase inicial.

Tabela 2 - Índices de velocidade de desenvolvimento ${ }^{(*)}$ de plantas de arroz nos estádios V1 (IVD V1), V2 (IVD V2) e V3 (IVD V3), em ambiente sem (SE) e com estresse (CE) por frio. Development speed index of rice plants at stages V1 (IVD V1), V (IVD V2), and V3 (IVD V3) in non stressed (SE) and cold stressed (CE) environments.

\begin{tabular}{lccccccccc}
\hline \multirow{2}{*}{ Cultivares } & \multicolumn{3}{c}{ IVD V1 } & \multicolumn{3}{c}{ IVD V2 } & \multicolumn{3}{c}{ IVD V3 } \\
\cline { 2 - 10 } & SE & CE & $\%$ & SE & CE & $\%$ & SE & CE & $\%$ \\
\hline Brilhante & $7,47 \mathrm{aA}$ & $3,59 \mathrm{aB}$ & 48 & $5,09 \mathrm{aA}$ & $2,26 \mathrm{aB}$ & 44 & $3,26 \mathrm{aA}$ & $1,34 \mathrm{aB}$ & 41 \\
SCSBRS TioTaka & $6,36 \mathrm{bA}$ & $1,98 \mathrm{bB}$ & 31 & $4,62 \mathrm{abA}$ & $1,42 \mathrm{bB}$ & 31 & $3,38 \mathrm{aA}$ & $0,78 \mathrm{bB}$ & 23 \\
BRS Querência & $6,09 \mathrm{bA}$ & $2,09 \mathrm{bB}$ & 34 & $4,43 \mathrm{bA}$ & $1,48 \mathrm{bB}$ & 34 & $3,06 \mathrm{aA}$ & $0,73 \mathrm{bB}$ & 24 \\
\hline CV (\%) & & 11,1 & & & 10,1 & & & 17,3 &
\end{tabular}

${ }^{(*)}$ Médias seguidas pela mesma letra maiúscula na linha e minúscula na coluna não diferem significativamente entre si, pelo teste de Tukey, a 5\% de probabilidade; CV - coeficiente de variação.

A partir do IVDi, foi possível calcular os índices de estresse e a média geométrica, que possibilitaram classificar as cultivares em relação à maior ou à menor tolerância ao estresse por frio (Tabela 3). Observou-se que, quanto maior o valor do índice de tolerância ao estresse por frio (ITS), maior foi o valor da média geométrica, sendo ambos diretamente proporcionais. Baseado nos cálculos de ITS e MG foi realizado o ranqueamento das cultivares, ao verificar que a Brilhante possui maior tolerância ao frio em relação à SCSBRS Tio Taka e BRS Querência. Esses resultados foram semelhantes àqueles visualizados na Tabela 2, indicando superioridade da cultivar Brilhante quanto ao IVDi, na condição de estresse por frio, em relação às demais. Os resultados dos índices em questão estão de acordo com os encontrados por BEVILACQUA et al. (2013), ao verificarem o desempenho da cultivar de arroz Brilhante quanto à tolerância ao frio para o comprimento radicular. Os autores constataram que a BriIhante apresentou maior tolerância ao frio para as avaliações de comprimento, seguidas das cultivares IRGS 422 CL, ecótipo 116 de arrozvermelho, enquanto a BRS6 Chuí apresentou menor desempenho nas avaliações.

A tolerância à seca em cevada cultivada e em silvestre foi avaliada por NAZARI \& PAKNIYAT (2010) utilizando também os índices de estresse. Os resultados obtidos por esses autores evidenciaram que a cevada silvestre apresentou índice de tolerância à seca superior à cevada cultivada, apesar do baixo rendimento, destacando a aptidão dessa primeira para ser usada em programas de melhoramento genético vegetal para a transferência de tal característica, em especial para as regiões onde existe limitação hídrica durante a safra. Já FLORIDO et al. (2009), em suas pesquisas, mostraram que os índices de tolerância ao estresse também podem ajudar em programas de melhoramento para permitir a verificação do desempenho de germoplasma sob condições ambientais adversas.

Neste trabalho, os índices de estresse também foram utilizados para avaliar o desem- 
penho das cultivares de arroz em cada estádio de desenvolvimento. A cultivar Brilhante apresentou resultados superiores para o índice de tolerância ao frio (ITS), baseado no índice de velocidade de desenvolvimento em cada estádio (V1, V2 e V3) (Tabela 4). A SCSBRS Tio Taka e BRS Querência mostraram desempenho semeIhante para o IVD no estádio V1, V2 e V3 para o índice de tolerância e suscetibilidade ao estresse por frio e uma redução decrescente para a média geométrica. $O$ ranqueamento dos genótipos foi baseado nos valores dos índices de tolerância ao estresse (ITS) e média geométrica (MG), em cada estádio de desenvolvimento apresentado na Tabela 4. O IVD dos genótipos nos estádios $\mathrm{V} 1$,V2 e V3 apresentaram ranqueamento semeIhante ao verificado no IVDi (Tabela 3 ).

Tabela 3 - Índice de tolerância ao estresse (ITS), de suscetibilidade ao estresse (ISS), média geométrica (MG) e "ranking" (R) de três cultivares de arroz irrigado, determinados pelo índice de velocidade de desenvolvimento incial (IVDi). Stress tolerance index (ITS), stress susceptibility (ISS), geometric mean $(\mathrm{MG})$, and ranking $(\mathrm{R})$ of three irrigated rice cultivars as determined by the initial development index (IVDi).

\begin{tabular}{llllc}
\hline Cultivares & ITS & ISS & MG & ${ }^{(*)} \mathrm{R}$ \\
\hline Brilhante & $0,7^{*}$ & 0,8 & 7,4 & 1 \\
SCSBRS TioTaka & 0,3 & 1,2 & 4,5 & 2 \\
BRSQuerência & 0,3 & 1,1 & 4,5 & 3 \\
\hline
\end{tabular}

${ }^{(*)} \mathrm{O}$ "ranking" (R) baseou-se no ITS e na MG.

Tabela 4 - Índices de tolerância ao estresse (ITS), de suscetibilidade ao estresse (ISS), médias geométricas (MG) e "ranking" de três genótipos de arroz irrigado, determinados pelo índice de velocidade de desenvolvimento dos estádios (IVD V1), V2 (IVD V2) e V3 (IVD V3). Stress tolerance index (ITS), stress suceptibilidade index (ISS), geometric mean (MG) and ranking of three irrigated rice genotypes determined by the speed index of stages V1 (IVD V1), V2 (IVD V2) and V3 (IVD V3).

\begin{tabular}{lllllllllll}
\hline \multirow{2}{*}{ Cultivares } & \multicolumn{3}{c}{ IVD V1 } & \multicolumn{3}{c}{ IVD V2 } & \multicolumn{3}{c}{ IVD V3 } & \multirow{2}{*}{${ }^{(*)} \mathrm{R}$} \\
\cline { 2 - 12 } & ITS & ISS & MG & ITS & ISS & MG & ITS & ISS & MG & \\
\hline Brilhante & $0,6^{*}$ & 0,8 & 5,2 & 0,5 & 0,9 & 3,4 & 0,4 & 0,8 & 2,5 & 1 \\
SCSBRS Tio Taka & 0,3 & 1,1 & 3,6 & 0,3 & 1,1 & 2,6 & 0,3 & 1,1 & 1,7 & 2 \\
BRS Querência & 0,3 & 1,1 & 3,6 & 0,3 & 1,1 & 2,6 & 0,2 & 1,1 & 2,4 & 3 \\
\hline$(*)$
\end{tabular}

${ }^{(*)} \mathrm{O}$ "ranking" (R) baseou-se nos valores dos ITS e na MG.

Os coeficientes de correlação linear de Pearson entre os índices de estresses e média geométrica, apresentados na Tabela 5, mostraram-se alta e positivamente correlacionados nas avaliações do desempenho das três cultivares determinadas pelo índice de velocidade de desenvolvimento inicial e pelo índice de velocidade de desenvolvimento nos estádios V1, V2 e V3. Esses resultados assemelham-se aos encontrados por BEVILACQUA et al. (2013), ao verificarem correlação positiva entre a $M G$ e o ITS e ao avaliarem o crescimento das plantas aos 7 e 14 dias, em quatro cultivares de arroz.

As três cultivares avaliadas apresentaram correlação negativa entre o índice de suscetibilidade (ISS) e a média geométrica (MG) e entre o índice de tolerância (ITS) e de suscetibilidade (ISS) determinados no estádio de desenvolvimento V3 (Tabela 5). A correlação entre os índices de estresses podem variar, dependendo do estádio de desenvolvimento inicial. Portanto, para que estudos desse tipo tenham aplicabilidade, é importante verificar a correlação dos índices de estresse em cada estádio de desenvolvimento inicial, pois a falta de correlação entre a tolerância ao frio num estádio e outro torna o melhoramento para essa característica bastante complicado quando o objetivo é incorporar tolerância em vários estádios (DATTA \& SIDDIQ, 1983). A análise de correlação apresentada neste estudo, baseado no IVD em V3, está de acordo com os resultados dos índices apresentados nas Tabelas 3 e 4 referentes à aplicação dos demais índices de estresse, pois à medida que o índice de tolerância e a média geométrica aumentam, o índice de suscetibilidade diminui. Essa correlação, inversamente proporcional apresentada entre os índices, pode ser utilizada para selecionar cultivares tolerantes ao frio.

No entanto, a dificuldade de selecionar cultivares tolerantes ao frio a campo levou à necessidade de se utilizar condições controladas para que, dessa forma, possa exercer-se controle sobre a intensidade e a duração da temperatura baixa. Além disso, a seleção para tolerância ao frio só será efetiva se a pressão de seleção apropriada for usada, o que torna o controle do ambiente de estresse crítico (BLUM, 1988).

Nesta avaliação, os índices de tolerância ao estresse (ITS) e a Média geométrica (MG) 
foram os índices mais eficazes para selecionar cultivares tolerantes ao frio. Os índices de estresses são ferramentas que podem auxiliar na seleção de cultivares tolerantes ao frio devido à variação da temperatura a campo, a presença de fatores bióticos e a interação com outros elementos climáticos que têm dificultado a correlação entre os resultados obtidos no ambiente controlado e no campo, limitando a aplicabilidade da seleção em condições controladas.

Tabela 5 - Correlação entre os índices de estresses e a média Geométrica de três cultivares de arroz determinados pelos índices de velocidade de desenvolvimento inicial (IVDi) e índices dos estádios V1 (IVD V1), V2 (IVD V2) e V3 (IVD V3). Correlation between the stress index and geometric mean of three rice cultivars determined by initial development index (IVDi) and stages index V1 (IVD V1), V2 (IVD V2) and V3 (IVD V3).

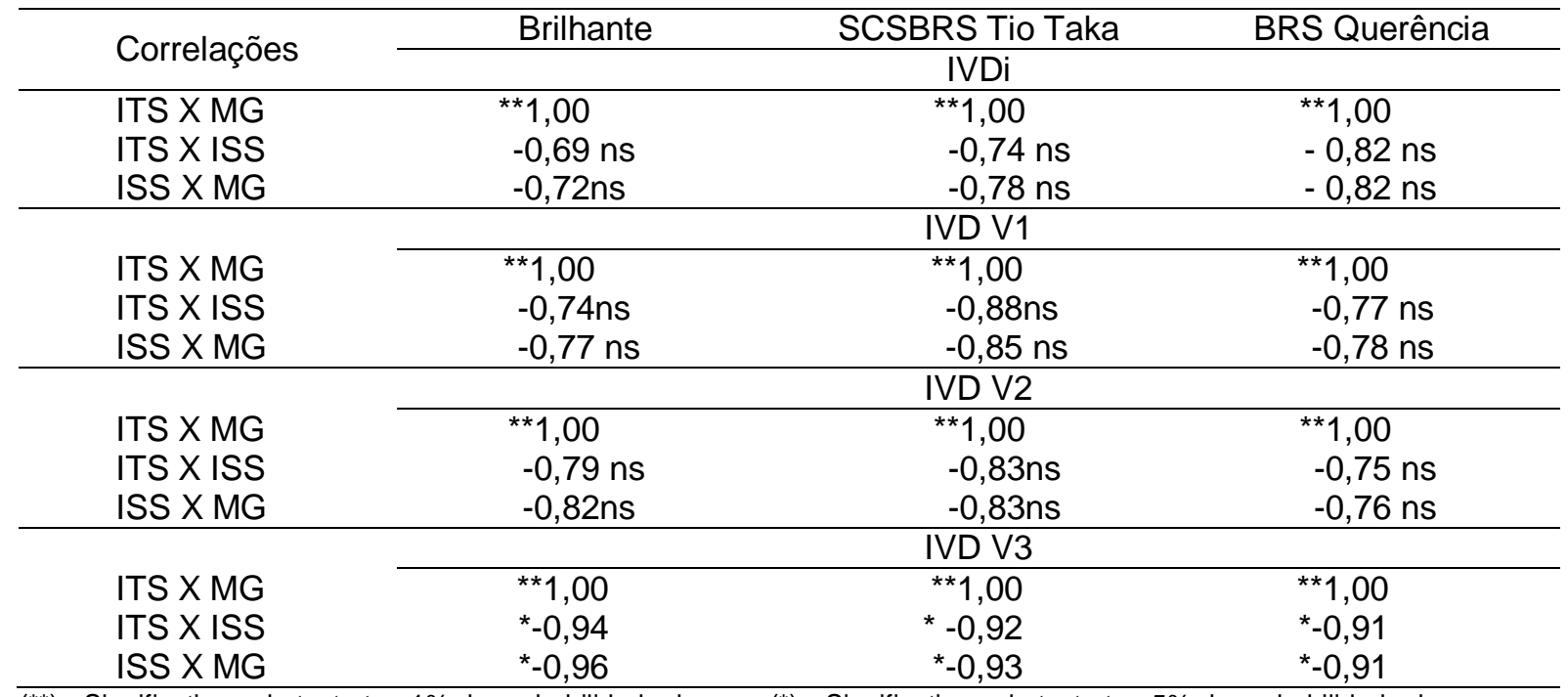

$\left(^{* *}\right)$ - Significativo pelo teste $\mathrm{t}$, a $1 \%$ de probabilidade de erro; $\left({ }^{*}\right)$ - Significativo pelo teste $\mathrm{t}$, a $5 \%$ de probabilidade de erro; $\mathrm{ns}$ não significativo pelo teste t; ITS - Índice de tolerância ao estresse; ISS - índice de suscetibilidade ao estresse; MG - Média Geométrica.

\section{Conclusões}

Os índices de estresse são eficientes para estimar a tolerância das cultivares em condições de baixa temperatura, destacando a superioridade da cultivar Brilhante nas fases iniciais de desenvolvimento (SO-V3) da cultura do arroz, no Rio Grande do Sul.

\section{Agradecimentos}

À Embrapa Clima Temperado, pelo apoio na realização da pesquisa, e à Coordenação de Aperfeiçoamento de Pessoal de Nível Superior (CAPES), pela concessão de suporte financeiro à pesquisa.

\section{Referências}

ARROZ IRRIGADO: recomendações técnicas da pesquisa para o Sul do Brasil / 28. Reunião Técnica da Cultura do Arroz Irrigado. Bento Gonçalves, RS; Porto Alegre: SOSBAI, 2010.188p.

BEVILACQUA, C. B.; MONZON, D. R.; VENSKE, E.; BASU, S.; ZIMMER, P. D. Application of Stress indices for low temperature and deep sowing stress screening of rice genotypes. Pakistan journal of biological Sciences,
Singapura, v.16, n.22, p.18-22, 2013.

BLUM, A. Plant breeding for stress environments. Boca Raton: CRC, 1988. cap. 5: Cold resistance, p.99-132.

CRUZ, R.P. Bases genéticas da tolerância ao frio em arroz (Oryza sativa L.). 2001. 155f. Tese (Doutorado em Fitotecnia). Universidade Federal do Rio Grande do Sul, Porto Alegre, 2001.

DATTA, D., SIDDIQ, E.A. Genetic analysis of cold tolerance at seedling phase in rice. Indian Journal of Genetics and Plant Breeding, New Delhi, v.43, n.3, p.345-349, 1983.

FERNANDEZ, C. G. J. Effective selection criteria for assessing plant stress tolerance. In: Adaptation of Food Crops to Temperature and Water Stress. Shanhua: AVRDC, 1993. p.257-270.

FISHER. R. A.; MAURER, R. Drought resistance in spring wheat cultivars. I Grain yield responses. Australian Journal of Agricultural Research, Collingwood, v.29, n.5, p.897-907, 1978.

FREITAS, T. F. S.; SILVA, P. R. F.; MARIOT, C. H. P.; MENEZES, V. G.; ANGHINONI, I.; BREDEMEIER, C.; VIEIRA, V. M. Produtividade de arroz irrigado e eficiência da adubação nitrogenada influenciadas pela época de semeadura. 
Revista Brasileira de Ciências do Solo, Viçosa, MG, v.32, n.6, p.2397-2405, 2008. FLORIDO, M.; ALVAREZ, M. M.; LARA, R. M.; PLANA, D.; SHAGARODSKY, T.; MOYA, C. Plant heat tolerance screening of ex situ preserved tomato (Solanum L. section Lycopersicum subsection Lycopersicum) germplam. Cultivos Tropicales, La Habana, v.30, n.4, p.39-47, 2009.

KENNEDY, P. G.; HIGGINS, L. M.; ROGERS, R. H.; WEBER, M. G. Colonizrtion-competition tradeoffs as a mechanism driving successional Dynamics in ectomycorrhizal fungal communities. PLoS ONE, San Francisco, v.6, n.9, p.1-10, 2011.

LARROSA, R. M.; MARCHESAN, E.; SILVA, L. S.;AVILA, L. A. Doses e épocas de aplicação de nitrogênio na suscetibilidade do arroz a temperatura baixa na fase reprodutiva. Ciência Rural, Santa Maria, v.39, n.4, p.992-997, 2009.

MAGUIRE, J.D. Speeds of germination-aid selection and evaluation for seedling emergence and vigor. Crop Science, Madison, v.2, n.2, p.176-177, 1962.

MARTINS, A. F.; VIEIRA, E. A.; KOPP, M. m.; LUZ, V.K.; CARVALHO, M.F.; BRANCO, J. S. C; CUZ, R. P.; CARVALHO, F. I. F.; OLIVEIRA, A. C. Caracterização de famílias mutantes de arroz para tolerância ao frio nos períodos vegetativo e reprodutivo. Bragantia, Campinas, v.66, n.2, p.227-233, 2007.
MERTZ, L. M.; HENNING, F. A.; SOARES, R. C.; BALDIGA, R. F.; PESKE, F. B.; MORAES, D. M. Alterações fisiológicas em sementes de arroz expostas ao frio na fase de germinação. Revista Brasileira de Sementes, Londrina, v.31, n.2, p.254-262, 2009.

NAZARI, L.; PAKNIYAT, H. Assessment of drought tolerance in barley genotypes. Journal of Applied Sciences, Shiraz, v.10, n.2, p.151-156, 2010.

STEINMETZ, S.; MATZENAUER, R.; MALUF, J. R. T.; FERREIRA, J. S. A. Frequência de temperatura do solo favorável à semeadura do arroz irrigado, no Estado do Rio Grande do Sul. Revista Brasileira de Agrometeorologia, Santa Maria, v.16, n.3, p.259-266, 2008.

STHAPIT, B.R.; WITCOMBE, J.R.; WILSON, J.M. Methods of selection for chilling tolerance in Nepalese rice by chlorophyll fluorescence analysis. Crop Science, Madison, v.35, n.1, p.90-94, 1995.

PORCH, T. G. Application of Stress Indices for heat tolerance Screening of common bean. Journal of Agronomy and Crop Science, Madison, v.192, n.5, p.390-394, 2006. 